\title{
Evaluation of low temperature degradation of 3Y-TZP in artificial saliva
}

\author{
María del Carmen Aragón Duarte ${ }^{1}$, Armando Reyes Rojas ${ }^{1}$, Hilda E. Esparza-Ponce ${ }^{1}$
}

1. Departamento de Física, Centro de Investigación en Materiales Avanzados S.C. (CIMAV), Miguel de Cervantes 120, Complejo Industrial, C.P. 31109, Chihuahua, México.

In the last years, $3 \mathrm{~mol} \%$ yttria-stabilized tetragonal zirconia polycystals (3Y-TZP) is consider as novel advanced ceramic material. It has been used in dentistry for restorations because its mechanical properties, biocompatibility, and its color similar to tooth color. [1] However, a disadvantage of the zirconia is the low temperature degradation or aging. Eventually, causing a decrease in mechanical properties and durability of the material. To determinate the integrity of the material normally accelerated aging test in steam water are made [2], simulating in a few hours what might happen in years of use. [3]

The present work evaluated the aging behavior of samples of 3Y-TZP sintered at $1400^{\circ} \mathrm{C}$, for one year under a solution of artificial saliva to oral conditions: temperature of $37^{\circ} \mathrm{C}$ and $\mathrm{pH} 6.8$

By XRD analysis was confirmed the existence of monoclinic phase after the aging. Using the Rietveld method phases were quantified. The hardness of the samples was determined using microhardness and the results obtained were compared with those reported in the literature that have been aged by the accelerated process.

The results of aging, show that degradation starts in the fourth month and reaches the maximum destabilization phase at 12 months with nearly $11 \%$ of monoclinic phase.

One of the most important results of this work is the comparison of degradation in artificial saliva compared to the accelerated aging test that are commonly used. The correlation is that five hours of accelerated aging corresponds to similar results from 1-2 months in artificial saliva. Because the test in situ allows the material is in contact with the solution 24 hours per day, the water has more opportunity to infiltrate between the grains, causing further phase transformation and thus is closer real oral conditions.

The results of the fracture toughness of the samples of 3Y-TZP, check what is written in the literature. Most transformation of tetragonal to monoclinic phase, most resistance to the crack from spreading because of the stress associated with volume expansion related to the phase change (figure 2). However, if the material continues deteriorate, there will come a time when the material will fracture.

References:

[1] PF Manicone, PR Iommeti and L Raffaelli, Journal of Dentistry 35 (2007), pp. 819-826.

[2] S Deville, L Gremillard, C Gerome and F Gilbert, Journal of Biomedical Materials Research Part B: 
Applied Biomaterials 72B (2005), pp. 239-245.

[3] ISO 13356 Implants for surgery - Ceramic materials based on yttria-stabilized tetragonal zirconia (Y-TZP)
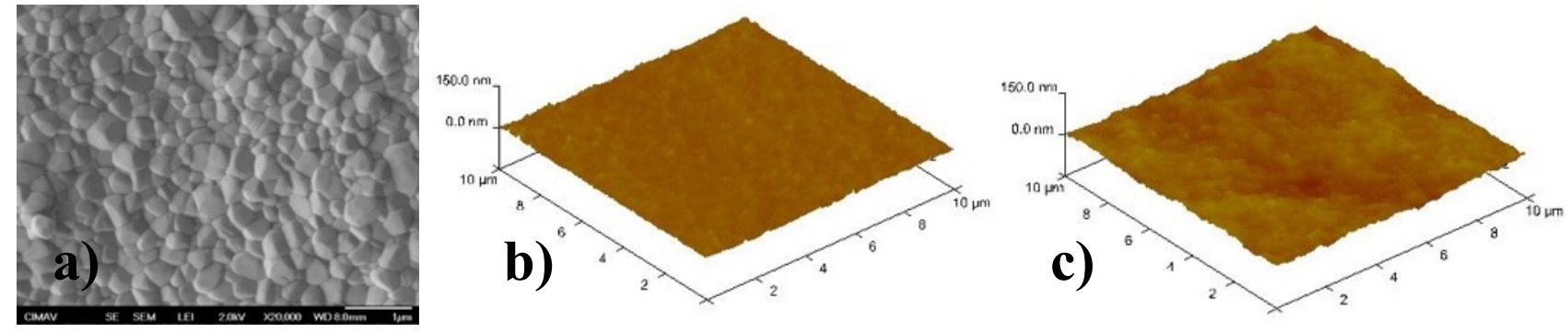

Figure 1. a) Scanning electron microscopy image of 3Y-TZP before aging. The grain size varies from about $100 \mathrm{~nm}$ to $500 \mathrm{~nm}$. b) Atomic force microscopy image before aging. The surface roughness is about $3 \mathrm{~nm}$. c) Atomic force microscopy image after 12 months of aging. The surface roughness increased to $6 \mathrm{~nm}$.

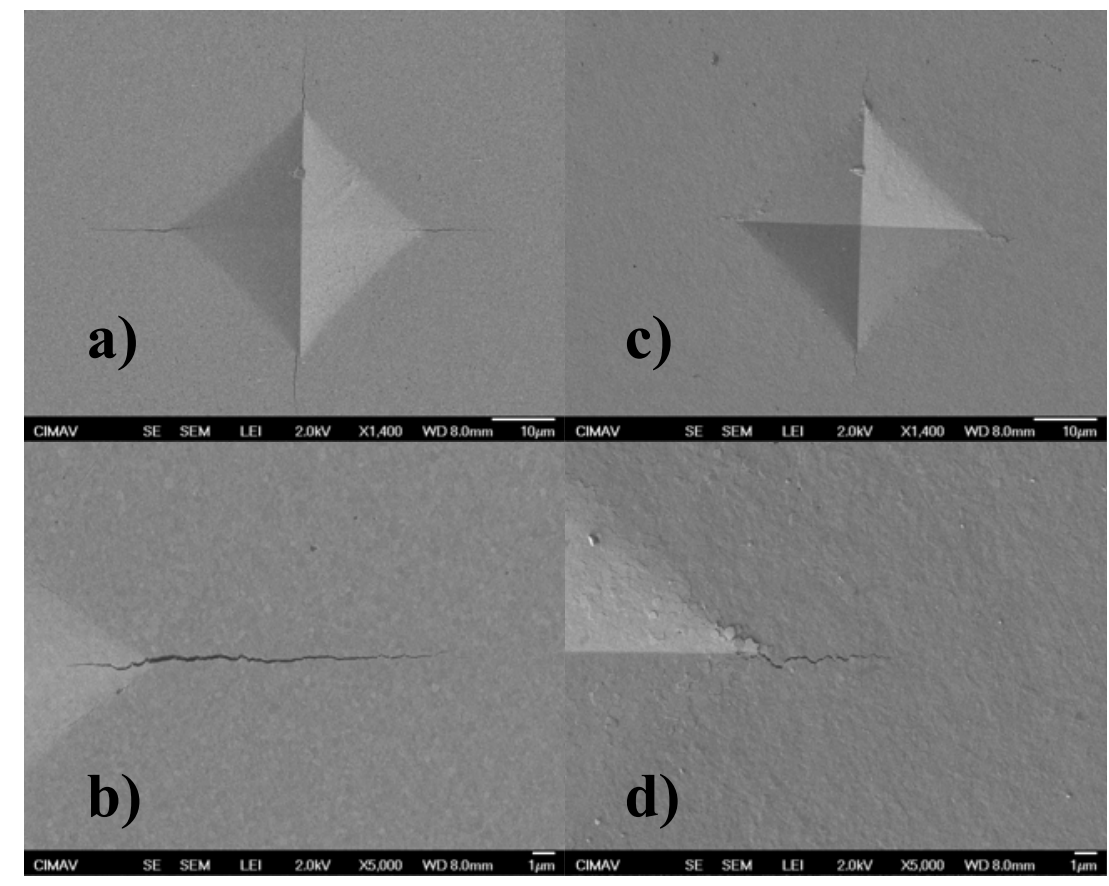

Figure 2. a) and b) Indentation of sample of 3Y-TZP before aging, c) and d) Indentation of sample of 3 Y-TZP after 12 months of aging. 